\title{
Land als Ressource, Boden und Landschaft: Materialität, Relationalität und neue Agrarfragen in der Politischen Ökologie
}

\author{
Daniel Münster and Julia Poerting \\ Cluster of Excellence "Asia and Europe in a Global Context", Heidelberg University, \\ 69115 Heidelberg, Germany
}

Correspondence to: Julia Poerting (julia.poerting@asia-europe.uni-heidelberg.de)

Received: 2 March 2016 - Revised: 8 August 2016 - Accepted: 15 August 2016 - Published: 17 October 2016

Kurzfassung. The Anthropocene reorients the agrarian question as an ecological question of planetary scale. Rather than resolving the inherent tension between political economy and the biophysical environment by moving political ecology closer to the natural sciences, we propose an active engagement with impulses from the environmental humanities and anthropological engagements with alternative ontologies. The relational political ecology of agriculture that we outline in this article draws on feminist science studies, multispecies ethnography, new materialism and critical geography. We show the relevance of a relational approach to agriculture as a natureculture entanglement by reviewing three conceptualisations of land in political ecology in relation to our anthropological research in South India (Münster) and geographical research in Northern Pakistan (Poerting). Notions of land as resource, land as soil and land as landscape respectively exemplify shifts in theoretical and political engagements with agriculture in the Anthropocene. A relational political ecology of agriculture incorporates these theoretical sensibilities and brings them in conversation with ontological politics of agro-ecological movements who respond to the variegated crises of the anthropocene. We suggest a perspective on agrarian landscape assemblages as coproduced by histories of capitalist transformations and the (affective) relations between humans, other species and materials.

\section{Einleitung}

Am Anfang war der Boden. Die ersten Forschungen, die den Ansatz der Politischen Ökologie geformt haben, beschäftigten sich mit Böden, genauer gesagt mit dem Zusammenhang von Bodenerosion und der politischen Ökonomie von Ländern des Globalen Südens. In ihrer vielzitierten Definition bestimmen die Geographen Harold Brookfield und Piers Blaikie den Ansatz wie folgt: „The phrase ,political ecology ' combines the concern of ecology and a broadly defined political economy. Together this encompasses the constantly shifting dialectic between society and land-based resources, and also within classes and groups within society itself" (Blaikie und Brookfield, 1987:17). In der Weiterentwicklung wurde Politische Ökologie zum Sammelbecken für kritische und angewandte Sozialwissenschaften, die sich mit Umweltproblemen im Kontext eines global expandieren- den extraktiven Kapitalismus beschäftigt (Peet et al., 2011). Als Folge des häufig politisch-ökonomischen Analyserahmens und der Nähe zu policy-Interventionen spricht Politische Ökologie eine Sprache, die biophysische Materialitäten und Lebensformen als umstrittene und kommodifizierte Ressourcen konzeptualisiert. Böden beispielsweise, die als komplexe Gefüge von Stoffwechselprozessen zwischen Menschen, Tieren, Mikroben, Mineralen und Pflanzen zu verstehen sind, werden häufig als Produktionseinheit Land behandelt. Die Reduktion dieser mehr-als-menschlichen Welten auf die Ressource Land stellt wichtige Fragen nach Umweltgerechtigkeit, Enteignungen und Privatisierungs- und Einhegungsprozessen, die zentrale Konfliktfelder im Zeitalter des Kapitals (Moore, 2015) darstellen. Allerdings hat die kritisch-realistische Perspektive auf Kapitalismus und Umwelt häufig einen modernistischen Dualismus von Gesellschaft (gedacht ohne Natur) und Natur (gedacht ohne Men- 
schen) reproduziert, den die Politische Ökologie angetreten war zu überwinden, oder zumindest zu verkomplizieren. In Anlehnung an Moores (2015:75 f.) Kritik der „metabolic rift"-Perspektive kann man allgemeiner feststellen, dass der konzeptuelle Apparat der Politischen Ökologie sich gewaltförmiger Abstraktionen oder ,violent abstractions“ (Moore, 2015:76) bedient, und etwa Land als produktive Ressource, als kartiertes Staatsgebiet, als privates Eigentum oder als vermessenes Katastergebiet versteht. Diese Konzepte entspringen einer Ontologie des Kapitals und stellen eher Teil des Problems als eine Quelle von Hoffnung dar. Für eine postkapitalistische Politische Ökologie des Anthropozäns scheint es uns angebracht, diesen modernistischen Konzepten von Land als Ressource solche zur Seite zu stellen, die die Relationalität, Materialität und Lebendigkeit von agrarischen MenschUmwelt-Beziehungen in den Vordergrund stellen.

In diesem Artikel diskutieren wir vorhandene Perspektiven auf Land, Landschaften und Böden in der Politischen Ökologie. Damit folgen wir einerseits der kritischen Frage Walkers nach dem Platz der Ökologie in der Politischen Ökologie (Walker, 2005), ohne jedoch seinem Ruf nach stärkerer Einbindung einer naturwissenschaftlichen Ökologie zu folgen, die umgekehrt eine Schwierigkeit hat, in hybriden Natur-Kultur-Räumen den Menschen anders als extern und als Bedrohung für Ökosysteme zu konzeptualisieren (siehe Kueffer, 2015; Nüsser, 2003). In unserer interdisziplinären Beschäftigung mit Land geht es uns daher um eine Verbindung von (im weitesten Sinne) marxistischen Perspektiven auf Zugang, Verteilung und Nutzung von Land mit Impulsen der Umweltgeisteswissenschaften (Environmental Humanities) - feministische Theorien zu Mensch-UmweltBeziehungen, Wissenschafts- und Technikforschung (STS) zu Soziomaterialitäten, Multispecies und umweltethnologische Forschung zu alternativen Ontologien - und mit agrarökologischen Ansätzen zu alternativen landwirtschaftlichen Praktiken.

Der Artikel basiert auf politisch-ökologischer Forschung zu Land und Landnutzung der Autoren in respektive Südindien und Nordpakistan. Daniel Münsters ethnologische Forschung beschäftigt sich einerseits mit der Politischen Ökologie von Agrarkrise, Bodendegradation und Bauernselbstmorden (Münster, 2015b, c) und andererseits mit alternativen Ontologien einer Naturlandbau-Bewegung (Münster, 2015a, 2016) in Südindien. Julia Poertings geographische Forschung beschäftigt sich mit der Einbindung pakistanischer Kleinbauern in globale Marktsysteme mittels Standards in der zertifizierten Biolandwirtschaft (Poerting, 2016) und in diesem Kontext mit Einhegungsprozessen von Land (Poerting, 2015). Die hier vorgestellten Überlegungen spiegeln den Stand unserer interdisziplinären Konversationen zwischen Humangeographie (Poerting) und Ethnologie
(Münster) im Rahmen der Nachwuchsforschergruppe ,Agrarian Alternatives“ an der Universität Heidelberg ${ }^{1}$ wider.

In einem ersten Schritt diskutieren wir für uns zentrale Konzepte einer zeitgemäßen Politischen Ökologie - Ontologie, Materialität, Relationalität, Neoliberalisierung. In den folgenden drei Kapiteln widmen wir uns jeweils einer Perspektive auf Land in der Politischen Ökologie und in Bezug auf unsere Forschung. Erstens diskutieren wir Land als Ressource, indem wir, anknüpfend an das Werk von Polanyi (1995 [1944]), Peluso und Lund (2011) und anderen, uns mit der Kommodifizierung von Land sowie den Prozessen seiner Einhegung, Privatisierung und Enteignung befassen. Über eine Beschäftigung mit neuen Agrarbewegungen, den sogenannten food sovereignty-Bewegungen, nähern wir uns zweitens der Frage von Land als Boden an. In diesen ländlichen Bewegungen rücken neben der Verteilungsfrage von Land zunehmend eine agrarökologische Kritik der industriellen Landwirtschaft und mögliche alternative Bodenontologien in den Fokus. Drittens beschäftigen wir uns mit möglichen Perspektiven auf Land als Landschaft. Dies beinhaltet zum einen die Kommodifizierung natürlicher und touristischer Landschaften und zum anderen eine holistische Perspektive auf komplexe, interdependente mehr-als-menschliche Nutzer von Boden und Landschaft. Davon ausgehend entwickeln wir aufbauend auf theoretischen Entwicklungen an der Schnittstelle von Geographie, Ethnologie und STS, eine relationale Politische Ökologie der Landwirtschaft.

\section{Eine relationale Politische Ökologie der Landwirtschaft (im Anthropozän)}

Die von Weichhart gestellte Frage wie man ,aus sozialwissenschaftlicher Perspektive sinnvoll mit der physischmateriellen Welt umgehen" (2003:19) könne, ist nicht neu für sozial- und geisteswissenschaftliche Forschung zu Natur und Umwelt. Die Politische Ökologie beschäftigt sich seit ihren Anfängen mit Fragestellungen über die Beziehungen und Wechselwirkungen von politischer Ökonomie und natürlicher Umwelt. Da sie sowohl von einer marxistischrealistischen Tradition beeinflusst als auch von einem Interesse an der biophysisch-materiellen Natur geleitet ist, wohnt ihr von Anfang an eine produktive Spannung inne: sie versteht die biophysische Umwelt sowohl als materiell gegeben, als auch als sprachlich-kulturell konstruiert und historisch-politisch produziert. In diesem Spannungsfeld formierten und formieren sich Ansätze, die sich in der For-

\footnotetext{
${ }^{1}$ Die interdisziplinäre Nachwuchsforschergruppe „Agrarian Alternatives: Agrarian Crisis, Global Concerns and the Contested Agro-ecological Futures in South Asia“ am Heidelberger Exzellenzcluster „Asien und Europa im globalen Kontext" beschäftigt sich mit alternativen Landwirtschaften in Südasien an der Schnittstelle von Geographie, Ethnologie und Wissenschafts- und Technikforschung. Für weitergehende Informationen siehe http://www.asia-europe.uni-heidelberg.de/en/research/ c-knowledge-systems/c15-agrarian-alternatives.html.
} 
schungspraxis niederschlagen. So beschäftigt sich politischökologische Forschung sowohl mit ökologischen Fragen von Biodiversität, Bodendegradation und Artensterben (Bennett, 2010; Altieri, 2002), mit konstruktivistischen Fragen nach der Kulturgebundenheit von Kategorien wie Wildnis, Natur und Landschaft (Braun, 2002; Escobar, 1999) als auch mit kritischen Fragen nach Ausbeutung, Gerechtigkeit und Verteilung (Lund, 2011; Bunker, 2005; Forsyth, 2008).

Eine krisenhafte und dringliche Sicht auf MenschUmwelt-Beziehungen im Zeitalter des Anthropozäns ${ }^{2}$ (Crutzen, 2002; Swanson et al., 2015; Gibson-Graham und Roelvink, 2010; Steffen et al., 2015; Latour, 2014) radikalisiert die Suche nach relationalen Ansätzen für die Politische Ökologie. Die Dualismen von Mensch-Natur, Natur-Kultur und Naturwissenschaft-Sozialwissenschaft lösen sich, beeinflusst durch Impulse aus STS, Ethnologie sowie feministischer und postkolonialer Theorie, in neuen materialistischen Ansätzen auf. Konzepte wie naturecultures (Haraway, 2003), socionature (Swyngedouw, 1999), Multinaturalismus (Latour, 2011; Viveiros de Castro, 2012), Hybridität (Whatmore, 2002), hybrides Kollektiv (Callon und Law, 1995) Koevolution (Pollan, 2002), double internality (Moore, 2015), boundary objects (Star und Griesemer, 1989), Akteursnetzwerk (Latour, 2005) und Intraaktionen (Barad, 2003) tragen in unterschiedlichen Paradigmen zu einem Überkommen des NaturKultur-Dualismus bei, indem sie das Augenmerk auf KoKonstitution und Relationalität legen. Eine relationale Politische Ökologie hat daher den Anspruch mehr als nur ein epistemologisches Projekt zu sein und eine Sprache zu finden für eine relationale Ontologie von mehr-als-menschlichen Welten.

Relationale Ansätze stehen im Zusammenhang mit einem neuerlichen Interesse an ontologischen Fragestellungen in der geistes- und sozialwissenschaftlichen Umweltforschung (Leff, 2015; Escobar, 2008; Descola, 2013; Viveiros de Castro, 2015; Kohn, 2015). Laut Viveiros de Castro ist die Allgegenwart des Begriffes Ontologie Ausdruck des Zeitgeistes in zahlreichen Disziplinen, die tiefgreifenden wissenschaftstheoretischen Wandel erfahren:

\footnotetext{
${ }^{2}$ Der Begriff des Anthropozäns geht auf den Chemiker Crutzen und den Biologen Stoermer zurück (2000), die damit ein neues geologisches Zeitalter vorschlagen, mit dem die tiefgreifenden Einflüsse des Menschen auf seine Umwelt hervorgehoben werden. In den Sozial- und Geisteswissenschaften benennt das Anthropozän neuere Ansatzpunkte für interdisziplinäre Mensch-UmweltForschung (Swanson et al., 2015). Der Begriff zieht unseres Erachtens nach berechtigte Kritik auf sich, da er von einem homogenen „Anthropos“ auszugehen scheint und, ohne auf Klassen und geographische Ungleichheiten einzugehen, die undifferenzierte Gattung „Mensch“ zum Verursacher der gegenwärtigen Krise erklärt (Moore, 2015:169-173). Wir verwenden ihn hier, um die Dringlichkeit gegenwärtiger Umweltzerstörung herauszustellen und auf die konzeptuelle Zentralität von Mensch-Umwelt-Beziehungen für notwendige neue Konversationen zwischen Natur- und Geisteswissenschaften hinzuweisen.
}

,[...] the exhaustion of the critical nomos that separated the phenomenon from the thing in itself, and the breaking apart of the hierarchical division of labour between natural and cultural sciences, as well as between pure (theoretical) reason and practical (moral) reason. But perhaps above all, it expresses the growing feeling that our own Modern ontology (singular) such as laid down by the scientific revolution of the 17th century not only was made largely obsolete by the scientific revolutions of the early 20th century, but that it also turned out to have disastrous consequences when considered from its business end, i.e. as an imperialist, colonialist, ethnocidal and ecocidal ,mode of production'. Ontology came to the fore precisely at the moment the ontological foundations of our civilisation - and the unquestioned cultural supremacy of the peoples who founded it - are seen as starting to crumble" (Viveiros de Castro, 2015:8 f.).

Für die Politische Ökologie von Land und Landwirtschaft lesen wir Viveiros de Castros Kritik der westlichen modernen Ontologie als Aufforderung, umweltgeisteswissenschaftliche Zugänge zur materiellen Umwelt zu entwickeln, die eine konzeptuelle Sprache jenseits von Positivismus und Reduktionismus sprechen. Viele Vertreter der sogenannten ontologischen Wende, insbesondere ihre ethnologischen Vertreter, sehen als Quelle für neue Ontologien vor allem nicht-westliche Wissenssysteme (La Cadena, 2015; Descola, 2013). Für unser Anliegen sind jedoch ebenso wichtig Kritiken an der modernen Ontologie als Ontologie des Kapitals. Die neomarxistisch-realistische Kritik (Harvey, 1996, 2001; Moore, 2011; Foster et al., 2010; Braun, 2006) an einer dualistischen Ontologie von Natur und Gesellschaft formuliert alternative Ontologien weniger in Bezug auf kulturelle Differenz als vielmehr durch den Verweis auf das, was Viveiros de Castro das „,business end“ der modernen Ontologie nennt: die desaströsen Konsequenzen eines Natur-Kultur-Dualismus für die Welt des Anthropozäns. Der Anspruch dieser Autoren ist es, kartesische Binaritäten in dialektischen Weltverständnissen von „capitalism as world ecology“ (Moore, 2011) oder ,double internalities“ (Moore, 2015) aufzulösen. Einen weiteren Beitrag zu der Politik der Ontologie sehen wir in feministischen Theorien zu Körperlichkeit und Mensch-Tier-Beziehungen (Haraway, 2008), alternativen Lebensformen (Gibson-Graham, 1996) und Mensch-Boden-Beziehungen (Puig de la Bellacasa, 2015). Diese neueren feministischen Ontologien wiederum stehen in regem Austausch mit Ansätzen aus STS (Hustak und Myers, 2013; Margulis, 1998; Kirksey und Helmreich, 2010; Ingram, 2011), die wir als vierten Beitrag zu einer zeitgemäßen Politischen Ökologie der Landwirtschaft vorschlagen.

Eine relationale Ontologie von Land und Landwirtschaft bedeutet für uns ein Hinterfragen von gängigen Skalen und Maßstäben sowie ein Anerkennen der Materialität der involvierten Böden, Menschen, Pflanzen und Mikroben. Der Annahme folgend, dass Menschen Teil relationaler Gefüge sind, wird das Verständnis von Materialität zentral (Good- 
man, 2001). Materie kann dann nicht als ,passiver, amorpher Stoff" (Folkers, 2013:23) verstanden werden, der erst durch menschliche Sprache oder Praxis Wert und Bedeutung erfährt, sondern muss einerseits relational und andererseits, evident im Kontext von Böden (Ingram, 2011), als aktiv konzeptualisiert werden. Innerhalb dieses neuen Materialismus (Dolphijn und van der Tuin, 2012; Coole und Frost, 2010) hat sich dementsprechend zum einen ein Forschungsstrang entwickelt, der, anknüpfend an Ideen der AkteurNetzwerk-Theorie, die Wirkmächtigkeit von Dingen basierend auf ihrer Relationalität in assemblages beschreibt (Bennett, 2010; Li, 2007). Zum anderen, und in teilweisem Widerspruch zur thing-power wie Bennett sie versteht, wonach alle Dinge vital sind, haben sich verschiedene Ansätze geformt, die sich vor allem mit tatsächlich lebendigen „Kreaturen“ (Kirksey und Helmreich, 2010:545) beschäftigen. Unter dem Überbegriff der Multispecies Ethnografie oder Mehrals-Menschlichen-Welten sammeln sich Ansätze, die, ähnlich der Idee einer assemblage, das gemeinsame Werden (Haraway, 2008) verschiedener Organismen im Gegensatz zu einem separatistischen Blick auf die Welt in den Vordergrund stellen (Kirksey, 2014).

Während wir grundsätzlich mit posthumanistischen Ansätzen übereinstimmen, dass gerade in der Landwirtschaft Menschen als immer schon eingebunden in relationale Gefüge zu verstehen sind, ist es uns wichtig, an die multiskalaren Krisen von Boden, Ernährung und Landwirtschaft im Anthropozän anzuknüpfen. Multispecies Assemblages in der Landwirtschaft - von mikrobiellen Symbiosen bis zu globalen Nahrungsketten - sind an die Geschichte und Geographie der Gegenwart anzubinden. Agrarische Sozionaturen sind in den letzten Jahrzehnten zunehmend neoliberale Naturen, d.h. vermittels environmental fixes (Castree, 2008) mit den Dynamiken des globalen Neoliberalismus ko-konstituiert: Natur und Natürlichkeit werden zur Ware (Arsel und Büscher, 2012), Naturschutz wird Marktgesetzen unterworfen (McAfee, 1999), landwirtschaftliche Technologien und Biologien werden den globalen betriebswirtschaftlichen Ansprüchen an Standardisierung, Produktivität und Erschwinglichkeit angepasst (Weis, 2010; Goodman, 2001; Friedmann, 2005). Landwirtschaftliche Nutzflächen unterliegen einem globalen Wettbewerb zwischen Tank, Trog und Teller, also dem Anbau von Energiepflanzen, Futterpflanzen und Nahrungspflanzen, mit signifikanten Auswirkungen auf bäuerliche Landschaften.

Eine Politische Ökologie von Land und Landwirtschaft im Anthropozän muss sich daher zum einen ontologischen Fragen stellen und zum anderen sehr heterogenen theoretischen, ethischen und politischen Sensibilitäten gerecht werden. Wir verstehen diesen Artikel als programmatischen Beitrag, der die Heterogenität dieser Ansätze nicht auflöst, sondern an konkrete empirische Anwendungsgebiete rückbindet. Wir wollen die oben dargestellten umweltgeisteswissenschaftlichen Impulse nutzen, um anhand von drei Perspektiven auf Land einen konzeptionellen Beitrag zu einer zeit- gemäßen Politischen Ökologie zu leisten. Dies spiegelt den Stand unserer Überlegungen im gemeinsamen Projekt wider, der neben der Anerkennung kritischer Perspektiven auf Agrarkrise und globale Agrofoodsysteme solche Arbeiten in den Vordergrund stellt, die aus Relationalität und Verbundenheit Hoffnung ableiten. Wir beginnen daher mit einem Überblick über den Stand der Forschung aus kritischer Perspektive, die sich mit Land als Ressource unter dem Gesichtspunkt der Verteilungsgerechtigkeit befasst.

\section{Politische Ökologie von Land als Ressource}

Nach Marx ist die „Expropriation des Landvolks von Grund und Boden“ (Marx, 1968 [1867]) in der ursprünglichen Akkumulation die Grundlage der kapitalistischen Produktionsweise:

„Historisch epochemachend in der Geschichte der ursprünglichen Akkumulation sind alle Umwälzungen, die der sich bildenden Kapitalistenklasse als Hebel dienen; vor allem aber die Momente, worin große Menschenmassen plötzlich und gewaltsam von ihren Subsistenzmitteln losgerissen und als vogelfreie Proletarier auf den Arbeitsmarkt geschleudert werden“(Marx, 1968 [1867]:744).

Forschung zur Enteignung ländlicher Bevölkerung (Lund, 2011; Levien, 2015; Hall, 2013) und der Einhegung von Allmenden (commons) (Peluso und Lund, 2011; Akram-Lodhi, 2007) hat nicht an Aktualität und Brisanz verloren. Im Gegensatz zur historischen Epoche, die Karl Marx beschreibt, wird im globalen Kapitalismus des 21. Jhd. die „frei“" gewordene Arbeit nicht in den industriellen Sektor absorbiert (Münster et al., 2015). In Ländern des globalen Südens entstehen in den Worten von Tania Li surplus populations: „They are $[\ldots]$, surplus' to the needs of capital, and not plausibly described as a labour reserve“" $(\mathrm{Li}, 2010: 66)$. David Harvey beschreibt mit seinem Konzept der accumulation by dispossession die Grenzräume des globalen Kapitalismus und die Prozesse, durch die Arbeit, Rohstoffe, aber auch billiges Land gewaltförmig in die globale Marktwirtschaft inkorporiert werden (Harvey, 2003:139). Diese Formen von Enteignung und Landnahme beinhalten stets die Verwandlung von Land als komplexes relationales Gefüge in Land als Ressource über Prozesse, die als Kommodifizierung, Privatisierung und Einhegung (enclosure) beschrieben werden. Politisch-ökologische Forschung zu Land und Landwirtschaft muss sich vorwerfen lassen, die Betonung auf die Gewalt der Aneignung und ihre materiellen Konsequenzen gelegt zu haben, jedoch in ihrer Behandlung von Land als Ressource die epistemischen Grundlagen dieser auch kategorialen Verwandlung selten hinterfragt zu haben (siehe jedoch Ouma, 2015). Für Amita Baviskar ist dies Grund, anstelle von Politischer Ökologie von Cultural Politics of Natural Resources zu sprechen:

„Political ecology has $[\ldots]$ assumed that the primary significance of natural resources resides in their material use 
value. A forest becomes a bone of contention because its trees represent timber, fodder or fuel, material values desired by different social groups. Cultural politics suggests that natural resources have value within a larger economy of signification which crucially shapes their modes of appropriation“ (Baviskar, 2003:5052).

Eine relationale Politische Ökologie müsste daher in ihrer Beschäftigung mit Verteilungspolitiken von Land den grundsätzlich fiktionalen Charakter von Land als Ressource und Ware stets mitdenken. Der Begriff der fiktiven Waren, neben Land auch Arbeit und Geld, wurde von Karl Polanyi eingeführt um aufzuzeigen, dass Land und Boden einerseits als relationale Gefüge in menschliche Institutionen eingebettet sind und andererseits keine vom Menschen produzierten Waren sind: „Was wir als Grund und Boden bezeichnen, ist ein mit den Lebensumständen des Menschen untrennbar verwobenes Stück Natur. Dieses Stück Natur herauszunehmen und einen Markt daraus zu machen, war vielleicht das absurdeste Unterfangen unserer Vorfahren“ (Polanyi, 1995 [1944]:243).

Als ein Beispiel für die Konsequenzen dieser Konzeptualisierung von Land als Ware und Ressource kann die kritische politisch-ökologische Forschung zu sogenannten land grabs vor allem im Globalen Süden gelten (McMichael, 2012). Diese Literatur, die die Vermarktung von kleinbäuerlichem und kommunalem Land durch private und staatliche Akteure problematisiert, ist sehr nützlich in ihrer Kritik an den Enteignungslogiken dieses neoliberalen Entwicklungsmodells, beispielsweise präsentiert im ambivalenten Weltbank-Report von 2011 (Deininger und Byerlee, 2011). Dieser Bericht weist zum einen auf die Risiken für kleinbäuerliche Landwirtschaft hin, die mit diesen Investitionen einhergehen. Zum anderen behauptet er, dass mit adäquaten Vertragsbedingungen gute und faire Voraussetzungen für eine wirtschaftliche Entwicklung der betroffenen Region geschaffen werden. Politisch-ökologische Forschung hat nicht nur die Ambivalenz dieser Empfehlungen kritisiert, sondern auch anhand vieler empirischer Beispiele gezeigt, dass diese großräumigen sogenannten land deals oder land acquisitions weder zu Ernährungssicherheit, noch zu Armutsreduktion oder ökonomischer Entwicklung beigetragen haben (Akram-Lodhi, 2012; Borras et al., 2011; Hall, 2011). Gleichzeitig hat sie in ihrer Analyse jedoch häufig die Kategorien dieser neoliberalen Entwicklungsmodelle, Land als Ressource und Land als Ware, übernommen und operiert mit strukturalistischen „black boxes“, wie Finanzialisierung (Ouma, 2014). Damit trägt sie zu einer Verfestigung der epistemischen Gewalt einer Ontologie des Kapitals bei (McMichael, 2014b) und negiert eine relationale, materielle Ontologie von Land als etwas, das mehr als eine fiktive Ware ist. Ähnlich argumentiert auch de Schutter, der den - akademischen wie aktivistischen - Kritikern von land grabs vorwirft, sich zu sehr auf deren Mechanismen zu fokussieren. Er sagt: ,we have forgotten the cultural significance of land, and we reduce land to its productive elements - we treat it as a commodity, when it means social status and a lifeline for the poorest rural hou- seholds" (de Schutter, 2011:274). Seiner Meinung nach beschäftigen sich sowohl Forscher als auch internationale Akteure in der Entwicklungszusammenarbeit zu viel mit der Frage, wie große Investitionen in Land derart gestaltet werden können, dass sie zu Ernährungssicherung, Armutsreduktion und ökonomischer Entwicklung beitragen, und zu wenig damit, die kategoriale Gewalt, die land grabs beinhalten, grundsätzlich in Frage zu stellen (de Schutter, 2011). Seine Kritik ist gerechtfertigt, vernachlässigt aber ebenfalls die Materialität von Land und Boden als komplexes mehr-alsmenschliches relationales Gefüge.

In Julia Poertings Forschung zu zertifizierter Biolandwirtschaft in Nordpakistan stellen die Kommodifizierung und Einhegung von Land zentrale Konfliktpunkte zwischen Bauern und Exporteuren dar. In der Region Baltistan verkaufen Kleinbauern ihre Produkte (u.a. Sanddorn, Aprikosenkerne, Walnüsse, Mandeln, Maulbeeren) an Exportfirmen, die auch die jährlichen Zertifizierungskosten tragen. Baltistan liegt schwer zugänglich im Hochgebirge des Karakorum, was für die Exportfirmen eine Herausforderung nicht nur bei der Organisation der Zertifizierung, sondern auch in der Lagerung, der Weiterverarbeitung und dem Transport der Produkte darstellt. Der Zugang zu Land für die Zucht neuer Obstsorten sowie den Bau von Trocknungstunneln und Lagerhallen ist daher wesentlich. Ein Exporteur aus dem Tiefland Pakistans hat aus diesem Grund im Jahr 2012 begonnen, in verschiedenen Dörfern privates Land von den Kleinbauern zu pachten. Diese kleinflächigen Landpachten an einen Außenseiter stellen ein Novum in Baltistan dar. Schmidt (2004) stellt in einem Artikel zu der Nutzung von Privat- und Allmenderessourcen in Shigar in Baltistan fest: „In all lease types in Shigar the relationship between land owner and tenant goes beyond the pure lease contract. [...] In general, rent contracts can be interpreted as an instrument to integrate landless and poor households socially and economically" (Schmidt, 2004:324). Ackerbau und Tierhaltung sind in Baltistan also in ein komplexes soziales und ökologisches Gefüge eingebunden. Die Nutzung von Land, der Erhalt der Bodenfruchtbarkeit und die Pflege der Obstgärten sind eng verknüpft mit dörflichen Institutionen, dem Wassermanagement, der Pflege der Bewässerungskanäle und kommunalen Weidepraktiken. Über die kürzlich geschlossenen Pachtverträge sichern die Verpächter, einzelne Kleinbauern, dem Pächter, Inhaber einer Exportfirma, die Rechte zur Nutzung einzelner Felder entweder für den Obst- und Pflanzenbau oder zum Errichten von Trocknungseinheiten für zwanzig Jahre zu. Der Pächter selbst ist allerdings, abgesehen von wenigen Besuchen im Jahr, nicht anwesend und der Verpächter und ein paar Arbeiter sind zuständig für alle anfallenden Arbeiten. Die Kontrolle über das Land ändert sich also nicht de jure, sondern de facto (Peluso und Lund, 2011) und die beschriebenen Dynamiken stellen somit sowohl eine Kommodifizierung als auch einen Einhegungsprozess von Land dar. Die Kommodifizierung erfolgt über den kommerziellen Pachtvertrag mit der Exportfirma. Wenn der Exporteur über das gepachtete Land 
spricht, benutzt er eine Sprache von Land als Ressource, die gleichzeitig eine „ontologische Rekonfiguration“ (Ouma, 2015:139) produziert, die Böden dem Markt begreiflich machen: Er beschreibt die Größe des jeweiligen Feldes und erklärt die Wertsteigerung durch die Pflanzung neuer Hochertragssorten. Im Gegensatz dazu bleibt das Land für die Kleinbauern, die das Land verpachten, weiterhin eingebettet in die komplexen sozialen und ökologischen Zusammenhänge traditionaler Rechte und Pflichten der Landnutzung. Durch Biozertifizierung erhält das Land seinen Charakter als fiktive Ware und wird auf seine produktiven Elemente reduziert. Im Kontext von langfristigen Pachtverträgen erfolgt auch eine Art Einhegung, da der Einfluss auf die Landnutzung durch lokale Bevölkerungen eingeschränkt wird. Dies kann man mit Peluso und Lund als „,new form of enclosure“ (2011:672) verstehen, weil hier keine direkte Enteignung und sichtbare Einzäunung erfolgen, sondern zunächst nur die Kontrolle über bestimmte Felder gemeint ist. Neue Einhegungen extrahieren dieses Land aus einem komplexen sozialen Gefüge und verwandeln Land in eine Ressource, dessen entlegene „Ursprünglichkeit“ es wiederum zu einem Faktor der Vermarktung von Natur im globalen Biodiskurs macht. In der Forschung zu Land in Baltistan müssen solche kategoriale Verwandlungen und neoliberalen Aneignungen also mitgedacht werden.

Eine relationale Politische Ökologie von Land als Resource steht also sowohl vor konzeptionellen als auch methodischen Herausforderungen. Konzeptionell muss sie die Geopolitik des Wissens (Mignolo, 2002) um Land und Landnutzungsverhältnisse in die Theoriebildung mit einbeziehen. Dabei spielt eine Behandlung von Land als Ressource natürlich nicht ausschließlich den Logiken der Enteignung und Vermarktung in die Hände, sondern kann, etwa im Kontext von Landreformen, auch einer demokratischen Diskussion um Verteilungsgerechtigkeit dienlich sein. Methodische Herausforderungen bestehen sowohl „im Feld“ als auch in der Analyse der Daten, wobei die verwendeten Begriffe und Kategorien reflektiert werden müssen. Vor dem Hintergrund postkolonialer Kritik an der Dominanz westlicher Wissenssysteme sprechen wir hierbei, im Anschluss an Marisol de la Cadena, ethnographischer Forschung eine wichtige Rolle zu: ,$[\ldots]$ the idea that differences can connect rather than separate would suggest an anthropological practice that acknowledges the difference between the world of the anthropologist and the world of others, and dwells on such differences because they are the connections that enable ethnographic conversations" (La Cadena, 2015:27). In der ethnographischen Arbeit begegnet Politischen Ökologen Land nicht nur als Ressource, als messbare, handelbare, übertragbare Einheiten, sondern auch als konkreter, greifbarer und lebendiger Boden. Die Ethnographie des Nicht-menschlichen erfordert neben dem Repertoire der umweltgeisteswissenschaftlichen Methoden eine ernsthafte Auseinandersetzung mit Naturwissenschaften, die nicht ausschließlich an Kritik sondern auch an Dialog und Kollaboration interessiert ist. Wie Engel-
Di Mauro kritisiert, hat die Marxistische Politische Ökologie, trotz ihres Bekenntnisses zu einer Gesellschaft-Natur Dialektik, keinen adäquaten Zugang zur Belebtheit von agrarischen Böden gefunden. Laut Engel Di-Mauro geht dies bereits auf Marx, Bodenkunde zurück: „Marx's notions of soils as inert, inorganic, malleable chemical input-output boxes, without history or dynamic of their own, stand in contrast to his dialectical approach“ (2014:141). Nach Schneider und McMichael (2010) sollten gegenwärtige sozial-ökologische Theorien noch stärker die Begrenztheit der Bodenkunde des 19. Jahrhunderts zur Kenntnis nehmen, die sich in „Marx's Ecology“ (Foster, 2000) wiederspiegelt, und sich darum bemühen, einen prozessualen Begriff der Bodenfruchtbarkeit zu entwickeln, der auch die Möglichkeit agrarökologischen „Reparierens“ von Böden zulässt. Im Sinne einer zeitgemäBen Politischen Ökologie der Landwirtschaft wenden wir uns im folgenden Kapitel Perspektiven auf die Materialiät, Nutzung und Ontologien von Land als Boden zu.

\section{Politische Ökologie von Land als Boden}

Für Karl Polanyi spielt sich der gesellschaftliche Prozess in der Marktgesellschaft in einer Doppelbewegung ab, wo neben die Kommodifizierung von Land unter marktwirtschaftlichen Bedingungen notwendigerweise Gegenbewegungen treten. Diese Gegenbewegungen (Polanyi, 1995 [1944]:112) hätten das Ziel (ländliche) Gesellschaften vor den Verwerfungen des Marktes zu schützen. Während sich historische soziale Bewegungen im ländlichen Raum des globalen Südens überwiegend um den Zugang zu und die Verteilung von Land drehten, lässt sich seit einigen Jahrzehnten eine Hinwendung zu argarökologischen Problemstellungen in ländlichen Bewegungen beobachten. Unter dem Sammelbegriff der „Food Sovereignty Movements“ (Agarwal, 2014; Fairbain, 2010; Jansen, 2014) werden in der Literatur heterogene Bewegungen beschrieben, die indigene Bewegungen, Landlosenbewegungen und Kleinbauernbewegungen umfassen (Meek, 2014). Die Hinwendung zu agrarökologischen Fragestellungen in diesen neuen Doppelbewegungen gründet auf einer Kritik an den ökologischen Verwerfungen von Grüner Revolution und globalisierter industrieller Landwirtschaft. Food Sovereignty Bewegungen wie La Vía Campesina (Martínez-Torres und Rosset, 2014) bündeln ihre Kritik an der Prekarisierung kleinbäuerlicher Landwirtschaft in einer Infragestellung der grundsätzlichen Paradigmen und Ontologien kapitalistisch-industrieller Landwirtschaft: „Agroecology is one of the central arenas in which radical grassroots movements are actively constructing an alternative value system“ (Meek, 2014:48). Wesentliche Kritikpunkte dieser transnationalen Bewegungen sind die Enteignungslogiken von landwirtschaftlichen Modernisierungsparadigmen, die auf großflächige effiziente Landnutzung, Mechanisierung und Chemikalisierung setzen und der damit einhergehende Verlust von Biodiversität und Autonomie. Mit ihrer 
Kritik und alternativen Praktiken formulieren food sovereignty Bewegungen und ihre akademischen Verteidiger (McMichael, 2014a; Holt-Giménez und Altieri, 2012; Edelman, 2014; Patel, 2009) eine alternative Ontologie der Landwirtschaft: „Food sovereignty is [...] ultimately about an ontological contest between distinct visions regarding agriculture: as an economic sector with producing units employing a short-term market calculus, or a landscape inhabited by farmers/pastoralists/fisherfolk geared to sustainable ecological relations“" (McMichael, 2014a:952).

Agrarökologische Bewegungen, wie die Zero Budget Natural Farming (ZBNF) Bewegung in Südindien (Münster, 2015a, 2016), widersprechen zahlreichen Paradigmen und agronomischen Prinzipien der Grünen Revolution, zentral darunter erscheint uns ihre Kritik an der industriellen Ontologie des Bodens als unbelebtes, defizitäres Substrat. In neueren Agrarbewegungen verschiebt sich also auch der Diskurs von Land als Ressource zu Land als Boden. Agrarökologisch informierte Bodenontologien überlagern sich in diesen Bewegungen mit traditionalen Ontologien von belebtem, vitalem Boden. Im Gegensatz zu den produktivistischen Perspektiven der klassischen politischen Ökologie, in der Bodendegradation als Effekt von Armut und Übernutzung analysiert wird, stellen food sovereignty Bewegungen die Pluralität agronomischer Paradigmen in den Vordergrund. Neben die Kritik struktureller Enteignungslogiken modernisierter Landwirtschaft wird die Kritik der (westlichen, kapitalistischen, corporate) Agrarwissenschaft (Levins und Lewontin, 1985; Goodman, 2001), ihrer „toxischen Pflanzen“ (Shiva et al., 1999) und zerstörten Böden gestellt.

Die alternative Ontologie der südindischen ZBNF Bewegung entwickelte sich im Kontext regionaler Agrarkrisen, die Kleinbauern Südindiens seit den späten 1990er Jahren heimsuchen. Im Rückblick machen viele Bauern der Region Wayanad, in der Daniel Münster seit 2008 Feldforschung betreibt, die Substanzen, Technologien, Biologien und Prinzipien der Grünen Revolution für die Degradation ihrer Böden, den Ausfall ihrer Ernten, ihre Abhängigkeit vom Weltmarkt und in letzter Konsequenz für die Epidemie von Bauernselbstmorden verantwortlich (Münster, 2015b). Wie Bauern immer wieder betonen, haben sie ihre Böden einerseits durch den übermäßigen Gebrauch von petrochemischen Düngern und Bioziden und andererseits durch die Umstellung auf Monokulturen zerstört. Anhänger der ZBNF Bewegung berichten, wie sie neu gelernt haben, Bodenfruchtbarkeit unter Ausnutzung mikrobiologischer Prozesse und den metabolischen Beziehungen zwischen Menschen, Kühen, Pflanzen und Mikroorganismen wiederaufzubauen und langsam und sorgfältig zu pflegen. Die Kritik dieser Bewegung an der Grünen Revolution, die vor allem von ihrem Gründer Subhash Palekar in Büchern (z.B. Palekar, 2010), im Internet und auf großen mehrtägigen Informationsveranstaltungen formuliert wird, postuliert eine explizite ontologische Differenz zwischen einem mechanistischen westlichen Verständnis von Boden und einem ,indischen“ Wissen über Boden, von den
Veden hergeleitet und in einer eigenen Begrifflichkeit formuliert, welches Böden als fruchtbar, Leben spendend und als „ocean of endless nutrients“ (Palekar, 2010:98) versteht. Mit den Worten von Heather Paxson gesprochen praktizieren ZBNF Bauern eine Art von „Mikrobiopolitik“ (Paxson, 2014), in der sie der Aktivität von Mikroorganismen neue Wertschätzung zukommen lassen. Paxson führt den Begriff Mikrobiopolitik ein: ,to call attention to the fact that dissent over how to live with microorganisms reflects disagreement about how humans ought to live with one another" (Paxson, 2014:115). Durch ihre Wertschätzung der mikrobiellen Aktivität in selbstgebrauten Fermenten von Kuhprodukten (Urin, Dung) stellen sie sich gegen die antimikrobiellen Prinzipien (Ingram, 2011) der modernen input-intensiven Landwirtschaft. Diese kleine Gruppe radikaler Landwirte betont die symbiotische Interdependenz von Mensch und nichtmenschlichen Akteuren (Margulis, 1998) in der Landwirtschaft und die Bedeutung der Aktivität unsichtbarer Bodenbewohner. Landwirtschaft selbst wandelt sich in dieser Bewegung von einer betriebswirtschaftlichen Reduktion auf Produktivität und Profit hin zu einem Lebensstil, in dem Fürsorge, $\mathrm{Affekt}^{3}$ und Relationalität im Vordergrund stehen.

In der Perspektive auf Land als Boden zeigt sich also eine Konvergenz von alternativen Praktiken, der ontologischen Politik von agrarökologischen Bewegungen und neueren Forschungen in Biologie (Margulis, 1998) und Agrarökologie (Altieri, 2002; Vandermeer, 1996), wobei letztere von der feministischen Wissenschafts- und Technikforschung in fruchtbare Konzepte für eine postkapitalistische relationale Ontologie übertragen werden (Puig de la Bellacasa, 2015; Tsing, 2014; Haraway, 2008; Stengers, 2010). An der Schnittstelle von (teilweise marginalisierten) Lebenswissenschaften, STS und den Diskursen indigener und alternativer Praktiker entsteht eine neue Sprache, die wir für richtungsweisend für eine nicht-reduktionistische Politische Ökologie der Landwirtschaft halten. Ökologie und biophysische Prozesse erfahren eine neue Aufmerksamkeit in der politischen Ökologie (Engel-Di Mauro, 2014) indem Landbau konsequent als ein mehr-als-menschliches Unterfangen beschrieben wird, in dem Menschen mit zahllosen sichtbaren und unsichtbaren Arten symbiotisch und interdependent in einem „entanglement of life-propagating relations“ (Ly-

\footnotetext{
${ }^{3}$ Mit dem Begriff Affekt verweisen wir auf neuere Forschung zu affektiven Ökologien, wie sie etwa 2015 auf der Tagung der American Association of Geographers in Chicago von Neera Singh, Daniel Münster und zahlreichen weiteren Forscherinnen und Forschern als eine politische Ökologie vorgestellt wurde, die, aufbauend auf dem Werk der Philosophen Spinoza, sowie Deleuze und Guattari [thousand plateaus], relationalen Ontologien und vitalen Materialitäten verpflichtet ist (Singh, 2013). „To the extent that affect is about how changes in one body (a nation, a habitat, a farmer, a monkey) result in transformations in other bodies, affective ecologies enable a nuanced understanding of complex relations with a focus on perpetual adjustments and motion." (Aitken und An, 2012:7).
} 
ons, 2014:212) verwoben sind. Neue Ontologien von Böden als lebendige interdependente multispecies Gemeinschaften (vgl. Puig de la Bellacasa, 2015:692) tragen aber nicht nur zu einem theoretisch nuancierterem Verständnis von Landwirtschaft im Sinne eines posthumanistischen Materialismus bei, sondern haben auch politisch-ökonomische Konsequenzen im Anthropozän. Komplexe Mensch-Boden-Tier-Pflanze Gefüge bedürfen Sorgfalt, Pflege und Aufmerksamkeit, die sich nicht über produktivistische Ansätze und fortschrittsorientierte Zeitskalen auflösen lassen. Die feministische Wissenschaftsphilosophin Maria Puig de la Bellacasa thematisiert agrarische Alterontologien in Bezug auf Zukunftsmodelle moderner Agrarwissenschaft: „Beyond science, foodweb models and scientific ideas of soil as living are explicitly made to speak for alternative soil care and human-soil relations, with implications for the dominant productionist futurity“ (Puig de la Bellacasa, 2015:14). Selbstverständlich steht auch in alternativen Landwirtschaften Produktion im Vordergrund: ,Yet here production is harnessed by good care rather than the contrary, and good care is tied to knowing and appreciating soil life. These practices speak of intensification, not so much intensification of production but rather of involvement with soils" (Puig de la Bellacasa, 2015:14).

Alternative Ontologien von Boden und Landwirtschaft, wie sie etwa von der südindischen ZBNF Bewegung formuliert und praktiziert werden, stellen eine besondere Herausforderung für die Politische Ökologie der Landwirtschaft dar. Einerseits sprechen sie deutlich zu neuen theoretischen Bemühungen um einen neuen relationalen Materialismus (Barad, 2003) und eine Anthropologie des Lebens (Kohn, 2013) und in diesem Kontext die Erschließung mehr-als-menschlicher Welten durch die Umweltgeisteswissenschaften. Andererseits läuft der Rekurs auf Ethnopedologie oder nichtwestliche Bodenkunde (Barrera-Bassols und Zinck, 2003) sowie nichtwissenschaftliche Weltzugänge und ihre Wissenschaftskritik einer einfachen Einbindung in Entwicklungsparadigmen und die Anknüpfung an Agrarwissenschaften entgegen. Nicht alle, auch progressive (Politischen) Ökologen werden bereit sein, solche Alterontologien (Hage, 2012) in ihre Theoriebildung einzubeziehen. Vor dem Hintergrund der Dringlichkeit neuer ökologischer Agrarfragen der Gegenwart sind wir der Meinung, dass sich die Politische Ökologie dem grundsätzlichen Hinterfragen ihres beharrlichen kartesischen Dualismus von Natur(wissenschaft) und Kultur(wissenschaft) nicht verwehren kann. Die Politische Ökologie von Land als Boden muss daher nicht weniger politisch sein als die Beschäftigung mit Land als Ressource. An die Stelle von Verteilungskämpfen, Einfriedungen und Enteignungen treten hier grundsätzliche Konflikte über nachhaltige Praktiken der Bodenbearbeitung und der Pflege von Biodiversität sowie die ontologische Politik affektiver Mensch-Boden-Beziehungen. In unserem Projekt, eine relationale politische Ökologie der Landwirtschaft zu skizzieren, haben wir uns zunächst mit dem Zugang zu landwirtschaftlichen Produktionsmitteln (Land als Ressource) und den bio- physischen Bedingungen des Pflanzenbaus (Land als Boden) beschäftigt. Im folgenden Kapitel wollen wir uns der Einbettung von Landwirtschaft in großräumigere Gefüge zuwenden und von Land als Landschaft sprechen.

\section{Politische Ökologie von Land als Landschaft}

Eine relationale Perspektive auf Landwirtschaft kann über Mensch-Boden-Beziehungen hinausgehen. Die Verwobenheit von Menschen mit ihren agrarischen Umwelten setzt sich auch über dem Maßstab des Feldes und der Produktionseinheit hinaus fort. Während eine relationale Perspektive auf Land als Boden das Hereinzoomen auf mikroskopische, mikrobische und biologische Prozesse beinhaltet, stößt die umgekehrte (herauszoomende) methodologische Blickrichtung auf Land als Landschaft. Damit ergibt sich neben den Perspektiven von Land als Boden und Land als Ressource eine weitere Betrachtungsebene der Politischen Ökologie von Land als Landschaft. Landschaft als epistemischer und ontologischer Begriff ruht auf einer problematischen Geschichte von Mensch-Natur-Dualismen, die sich sowohl im Geodeterminismus, im Anthropozentrismus des Landnutzungskonzeptes und in Varianten des Konstruktivismus zeigt (vgl. auch Weichhart, 2016). Der Geodeterminismus, in seinen rassistischen und modernisierungstheoretischen Varianten, geht von Landschaften als gegeben und wirkmächtig aus. Forschung zu Landnutzung untersucht im Gegensatz dazu, wie in das Land von Menschen eingegriffen wird. In beiden Fällen ist Land als neutral und materiell gegeben angesehen. Konstruktivistische (poststrukturalistische, diskurstheoretische) Auseinandersetzungen mit der Landschaft stellen diese „Gegebenheit“ grundsätzlich in Frage, und betrachten Landschaft(sbilder) als kulturelle und symbolische Produktionen. Determinismus und Gegebenheit wurde hier überwunden, allerdings auf Kosten eines ,Realitätsverlustes“ (Mattissek und Wiertz, 2014:157), der dazu neigt die Materialität und Lebendigkeit von Landschaft zu vernachlässigen.

Die relationale Perspektive auf Land als Landschaft, die wir vorschlagen, lehnt die klassische Unterscheidung von „Naturlandschaft“" und „Kulturlandschaft“ ab, nicht nur für agrarische Landschaften (vgl. auch Weichhart, 2003). (Agrar)landschaften verstehen wir immer sowohl als bewohnt und geformt von zahlreichen menschlichen und nichtmenschlichen Bewohnern (Barua, 2014), als auch als koproduziert durch Geschichten struktureller Veränderungen wie koloniale Plantagenwirtschaft oder globale frontiers (Eilenberg, 2014) der Energiepflanzenproduktion. Im Neoliberalismus werden Landschaften selber zu Waren - fiktiven Waren, mit Polanyi gesprochen. Die Kommodifizierung von agrarischen Landschaften erfolgt zum einen über den Tourismus (Münster und Münster, 2012), zum anderen über die Ausweisung von Naturschutzgebieten (Brockington und Duffy, 2010), sowie über die Romantisierung ihrer agrochemischen Unberührtheit in der Biolandwirtschaft (Poerting, 2016). Un- 
ser relationales Verständnis von Land als Landschaft schlägt die Synthese älterer Landschaftsbegriffe vor: Eine Perspektive auf die Materialität von Pflanzen, Gestein, Klima und Tieren, kombiniert mit einem Verständnis der Zentralität von Arbeit in Mensch-Umwelt-Beziehungen sowie der Bedeutung von materiell-semiotischen Konstruktionen - menschlichen und nicht-menschlichen.

Wir wollen also die Zentralität der symbiotischen und koproduktiven Prozesse in der Landwirtschaft in den Vordergrund stellen. Ein „Herauszoomen“ aus dem Boden und dem Feld ermöglicht einen Blick auf zusammenhängende Landschaften, die bewohnt (dwelling; Barua, 2014) und vielfältig gebraucht werden. Besonders im Anthropozän, in dem Landschaften nicht nur geschaffen und kommodifiziert, sondern auch in einem nie dagewesenen Maße verändert, verödet und zerstört werden, scheint uns eine nicht-anthropozentrische relationale Perspektive auf agrarische Landschaften angemessen. Im Verlauf unserer Forschungen über Agrarkrisen und ihre Alternativen hat sich für uns eine vergleichende Perspektive auf agrarische Landschaften und Regionen (Baltistan und Wayanad) als fruchtbare Grundlage für unsere interdisziplinären Konversationen über Politische Ökologie der Landwirtschaft erwiesen. In agrarischen Landschaften, wie dem südindischen Wayanad Distrikt, tritt Land einerseits als Ressource in Erscheinung. In den südindischen Westghats haben sich die Geschichten von Landnahmen, Landreformen und Waldschutzregimen in die Landschaft eingeschrieben. Die fragmentierte kleinbäuerliche Landschaft ist durch die Einbindung in Markt, Staatlichkeit und überstaatlichen Naturschutz geprägt. Verschiedene agrarische Praktiken, vom Nassreisanbau über kommerzielle Monokulturen, zu Naturlandbauformen, haben die Landschaft ebenso geprägt wie die beteiligten Kultivare (Arekanuss, Ingwer, Kaffee), Chemikalien (Biozide, Kunstdünger), Bodenmikroben (Pathogene und Symbionten) und Wildtiere des Wayanad Wildlife Sanctuary (Elephanten, Wildschweine, Tiger). Das Land des Wayanad Distriktes, das sich für ihre menschlichen Siedler von einer frontier der hoffnungsvollen Landnahme zu einer „blasted landscape“ (Kirksey et al., 2013) und einem hotspot der Agrarkrise gewandelt hat, ist somit andererseits als ein Gefüge aus multiplen und mehr-als-menschlichen Austauschbeziehungen, Metabolismen und Interdependenzen zu beschreiben. Historische Ontologien, vom kolonialen Blick auf vermeintlich leere unproduktive Landschaft bis zur postkolonialen Modernisierung der Landschaft in der Grünen Revolution, spielen in der materiell-semiotischen Realität dieses Landstriches eine ebenso bedeutende Rolle, wie die historischen Materialitäten von Lebewesen, Boden, Monsunregen und Chemikalien.

In unseren Forschungen zu Nordpakistan und Südindien zeigt sich der Wert einer holistischen Perspektive auf Agrarlandschaften. Sie ermöglicht eine Kombination aus einerseits kritisch-marxistischen Perspektiven auf Geschichten der Landbesitzverhältnisse, der Kapitalisierung von Landwirtschaft und der strukturellen Ursachen von Agrarkri- sen und andererseits Multispecies Perspektiven auf affektive Mensch-Boden-Tier-Beziehungen, die Wirkmächtigkeit von Substanzen und Materialitäten sowie ökologische Zusammenhänge. Wie unsere Forschung gezeigt hat, gehen alternative Umgangsformen von Kleinbauern mit ihrem Land, etwa in der indischen Zero Budget Natural Farming Bewegung, häufig von einer wahrgenommenen Krise aus, deren Bewältigung jedoch ein ebenso ökonomisches, praktisches wie epistemisch-ontologisches Projekt darstellt. Viele alternative Landwirtschaften beruhen darauf, agrarische Landschaft neu zu verstehen (ökologisch, biologisch, symbiotisch), neu zu bewohnen (affektiv, care-ful (Puig de la Bellacasa, 2015), response-able (Haraway, 2008)) und neu zu bewirtschaften (intensiv, vielfältig, resilient).

\section{Fazit}

Im Anthropozän stellt sich die Agrarfrage als ökologische Frage in planetarischem Maßstab neu. Der Politischen Ökologie fällt hierbei aufgrund ihrer historisch-materialistischen Perspektive auf globale Verteilungsfragen und dem Interesse an der biophysisch-materiellen Umwelt eine besondere Bedeutung zu. Hierbei gilt es besonders, an das produktive Spannungsverhältnis von sozialwissenschaftlicher Kritik und dem Wissen um ökologische Zusammenhänge in ihrer sozialökologischen Forschung anzuknüpfen. Während dieses Spannungsverhältnis herkömmlich darüber aufgelöst wurde, sozioökologische Forschung näher an die Paradigmen der Naturwissenschaften heranzuführen, scheint uns der umgekehrte Weg ebenso gangbar und sowohl politisch als auch philosophisch befriedigender. In unserer Skizze einer relationalen Politischen Ökologie der Landwirtschaft scheinen uns neuere Impulse aus den Umweltgeisteswissenschaften besonders zielführend. Die Beiträge aus feministischer und postkolonialer Wissenschafts- und Technikforschung, der Multispecies-Ethnographie, die ethnologische Forschung $\mathrm{zu}$ alternativen Ontologien sowie dialektisch orientierte kritische Geographen scheinen uns besonders hilfreich zu sein, die Untrennbarkeit und Koproduktion von hybriden Sozionaturen neu und innovativ $\mathrm{zu}$ bearbeiten, ohne dabei dem Reduktionismus und Positivismus vieler naturwissenschaftlicher Ansätze nachgeben zu müssen. Dies macht ein neuerliches Hinterfragen der persistierenden Arbeitsteilung zwischen den Natur- und Geisteswissenschaften, nach der Geisteswissenschaften auf Repräsentationalismus und sprachlichem Konstruktivismus beschränkt werden, und allein $\mathrm{Na}$ turwissenschaften sich zu Materialität, Leben und Ökologie kompetent äußern können, nötig.

Um mögliche neue Wege einer relationalen Politischen Ökologie aufzuzeigen, haben wir anhand eines zentralen Untersuchungsgegenstandes der Politischen Ökologie, Land, verschiedene Perspektiven dargestellt. Land scheint uns besonders geeignet, eine relationale Politische Ökologie zu exemplifizieren, da in der Kategorie Land sowohl ontologische 
Politiken am Werk sind, die gewaltförmigen Abstraktionen, die Land überhaupt erst in Eigentum beziehungsweise fiktive Ware verwandeln, als auch geisteswissenschaftliche Perspektiven auf die Materialität und Lebendigkeit der Böden, die die Relationalität ihrer Bewohner und Nutzer in den Vordergrund stellen. Land als Ware und Ressource bleibt, wenn auch fiktiv und epistemologisch unrein, eines der wichtigsten Gegenstände gegenwärtiger Konflikte und politischer Prozesse. Land hat aber auch eine konkrete Materialität, die in der Behandlung von Land als Boden in sozioökologische Forschung Einzug gehalten hat. Eine Beschäftigung mit Bodeneigenschaften und den vielfältigen Beziehungen, die Böden mit Menschen, Mikroben und Pflanzen eingehen, zeigt, dass hier neben neuerer mikrobiologischer Forschung auch situierte Ontologien von Leben, Fruchtbarkeit und Produktivität eine Rolle spielen. Kritische Perspektiven auf Landwirtschaft ergänzen daher Fragen des Zugangs zu und der Verteilung von Land mit Fragen der Politisierung der eingegangen Beziehungen mit dem Boden im Landbau. Gegenwärtige Agrarkrisen, ländliche Armut und die Prekarisierung kleinbäuerlicher Lebensweisen sind nicht allein auf die Politische Ökonomie globaler Agrarmärkte und Verteilungslogiken von Land zurückzuführen, sondern gründen auch in den Effekten „modernisierter“ Bodennutzung. Neue agrarökologische Bewegungen, wie die südindische Zero Budget Natural Farming Bewegungen, kritisieren gleichermaßen die verheerenden Effekte von Grünen Revolutionen, kapitalistischen Agrartechnologien und toxischen Bioziden. Aus diesen Bewegungen und ihrer Rezeption kristallisiert sich auch eine alternative postkapitalistische Ontologie, die auf affektive Mensch-Tier-Pflanzen-Boden-Beziehungen, Fürsorge, Symbiose und Interdependenz baut. Im Dialog mit widerständigen alternativen Ontologien kann es einer relationalen Politischen Ökologie gelingen den Blick noch konsequenter auf agrarische Landschaften als gleichzeitig diskursiv und materiell zu verstehen. Agrarische Landschaftsgefüge sind Koproduktionen von Geschichten struktureller kapitalistischer Verwerfungen und der Zusammenkunft einer Vielzahl an Lebewesen, Materialien und Chemikalien.

\section{Datenverfügbarkeit}

Dieser Artikel basiert auf ethnographischer Forschung beider Autoren. Die Daten sind nicht öffentlich zugänglich.

Danksagung. Wir bedanken uns bei Sören Becker und Antje Otto für die Einladung zu diesem Themenheft und bei dem anonymen Gutachter für die konstruktiven Kommentare. Die Forschungen zu diesem Artikel wurden vom Exzellenzcluster „Asien und Europa im globalen Kontext" der Universität Heidelberg, im Rahmen der Nachwuchsforschergruppe C15 (Agrarian Alternatives) finanziert.

Edited by: A. Otto

Reviewed by: one anonymous referee

\section{Literatur}

Agarwal, B.: Food sovereignty, food security and democratic choice: critical contradictions, difficult conciliations, J. Peasant Stud., 41, 1247-1268, 2014.

Aitken, S. and An, L.: Figured worlds: Environmental complexity and affective ecologies in Fanjingshan, China, Ecol. Model., 229, 5-15, 2012.

Akram-Lodhi, A. H.: Land, markets and neoliberal enclosure: An agrarian political economy perspective, Third World Q., 28, 1437-1456, 2007.

Akram-Lodhi, A. H.: Contextualising land grabbing: Contemporary land deals, the global subsistence crisis and the world food system, Can. J. Dev. Stud., 33, 119-142, 2012.

Altieri, M. A.: Agroecology: The science of natural resource management for poor farmers in marginal environments, Agr. Ecosyst. Environ., 93, 1-24, 2002.

Arsel, M. and Büscher, B.: Nature ${ }^{\mathrm{TM}}$ Inc.: Changes and Continuities in Neoliberal Conservation and Market-based Environmental Policy, Dev. Change, 43, 53-78, 2012.

Barad, K.: Posthumanist Performativity: Toward an Understanding of How Matter Comes to Matter, Signs, 28, 801-831, 2003.

Barrera-Bassols, N. and Zinck, J. A.: Ethnopedology: a worldwide view on the soil knowledge of local people, Geoderma, 111, 171195, 2003.

Barua, M.: Bio-geo-graphy: landscape, dwelling, and the political ecology of human-elephant relations, Environ. Plann. D, 32, 915-934, 2014.

Baviskar, A.: For a Cultural Politics of Natural Resources, Econ. Polit. Weekly, 38, 5051-5055, 2003.

Bennett, J.: Vibrant Matter: A political ecology of things, Duke University Press, Durham [NC], London, ISBN: 978-0-8223-46197, 2010.

Blaikie, P. M. and Brookfield, H. C.: Land degradation and society, Routledge, Oxon, ISBN: 1317411943, 1987.

Borras, S. M. J., Hall, R., Scoones, I., White, B., and Wolford, W.: Towards a better understanding of global land grabbing: An editorial introduction, J. Peasant Stud., 38, 209-216, 2011.

Braun, B.: The intemperate rainforest: Nature, culture, and power on Canada's west coast, University of Minnesota Press, Minneapolis, ISBN: 978-0816633991, 2002.

Braun, B.: Towards a New Earth and a New Humanity: Nature, Ontology, Politics, in: David Harvey: A Critical Reader, Herausgeber: Castree, N. und Gregory, D., Blackwell Publishers, Malden, MA, 191-223, 2006.

Brockington, D. and Duffy, R.: Capitalism and Conservation: The Production and Reproduction of Biodiversity Conservation, Antipode, 42, 469-484, 2010.

Bunker, S. G.: The poverty of resource extraction, in: New Directions in the Sociology of Global Development, Herausgeber: Buttel, F. H. und McMichael, P., Elsevier, Amsterdam, 211-227, 2005.

Callon, M. and Law, J.: Agency and the Hybrid Collectif, S. Atl. Quart., 94, 482-507, 1995.

Castree, N.: Neoliberalising nature: The logics of deregulation and reregulation, Environ. Plann. A, 40, 131-152, 2008.

Coole, D. H. and Frost, S. (Hrsg.): New materialisms: Ontology, agency, and politics, Duke University Press, Durham [NC], ISBN: 978-0822347729, 2010. 
Crutzen, P. and Stoermer, E.: The Anthropocene, Global Change Newsletter, 41, 17-18, 2000.

Crutzen, P.: Geology of Mankind, Nature, 415, 23, doi:10.1038/415023a, 2002.

Deininger, K. and Byerlee, D.: Rising Global Interest in Farmland: Can it Yield Sustainable and Equitable Benefits?, The World Bank, Washington, ISBN: 978-0971757585, 2011.

De la Cadena, M.: Earth beings: Ecologies of practice across Andean worlds, Duke University Press, Durham [NC], ISBN: 978$0822359449,2015$.

de Schutter, O.: How not to think of land-grabbing: Three critiques of large-scale investments in farmland, J. Peasant Stud., 38, 249279, 2011.

Descola, P.: Beyond nature and culture, University of Chicago Press, Chicago, ISBN: 978-0226145006, 2013.

Dolphijn, R. and van der Tuin, I.: New Materialism: Interviews and Cartographies, 1st ed., Open Humanities Press, ISBN: 978$1607852810,2012$.

Edelman, M.: Food sovereignty: forgotten genealogies and future regulatory challenges, J. Peasant Stud., 41, 959-978, 2014.

Eilenberg, M.: Frontier constellations: agrarian expansion and sovereignty on the Indonesian-Malaysian border, J. Peasant Stud., 41, 157-182, 2014.

Engel-Di Mauro, S.: Ecology, soils, and the left: An ecosocial approach, Palgrave Macmillan, New York, ISBN: 9781137350138, 2014.

Escobar, A.: After Nature: Steps to an Antiessentialist Political Ecology, Curr. Anthropol., 40, 1-30, 1999.

Escobar, A.: Territories of difference: Place, movements, life, redes, Duke University Press, Durham [NC], ISBN: 978-0822389439, 2008.

Fairbain, M.: Framing Resistance: International Food Regimes \& the Roots of Food Sovereignty, in: Food Sovereignty: Reconnecting Food, Nature \& Community, Herausgeber: Wittman, H., Desmarais, A. A., und Wiebe, N., Fernwood Publishing, Halifax, 15-44, 2010.

Folkers, A.: Was ist neu am neuen Materialismus?: Von der Praxis zum Ereignis, in: Critical Matter: Diskussionen eines neuen Materialismus, Herausgeber: Goll, T., Keil, D., und Telios, T., Edition Assemblage, Münster, 16-33, 2013.

Forsyth, T.: Political ecology and the epistemology of social justice, Geoforum, 39, 756-764, 2008.

Foster, J. B.: Marx's Ecology: Materialism and Nature, New York: Monthly Review Press, ISBN: 1583670122, 2000.

Foster, J. B., Clark, B., and York, R.: The ecological rift: Capitalism's war on the Earth, Monthly Review Press, New York, ISBN: 978-1583673881, 2010.

Friedmann, H.: From colonialism to green capitalism: Social movements and emergence of food regimes, in: New Directions in the Sociology of Global Development, Herausgeber: Buttel, F. H. und McMichael, P., Elsevier, Amsterdam, 227-265, 2005.

Gibson-Graham, J. K.: The end of capitalism (as we knew it): a feminist critique of political economy, Blackwell, Oxford, ISBN: 978-0816648054, 1996.

Gibson-Graham, J. K. and Roelvink, G.: An Economic Ethics for the Anthropocene, in: The Point is to Change It: Geographies of Hope and Survival in an Age of Crisis, Herausgeber: Castree, N., Chatterton, P., Heynen, N., Larner, W., und Wright, M. W.,
Wiley-Blackwell, Chichester, West Sussex, UK, Malden, MA, 320-347, 2010.

Goodman, D.: Ontology Matters: The relational materiality of nature and agro-food studies, Sociol. Ruralis, 41, 182-200, 2001.

Hage, G.: Critical anthropological thought and the radical political imaginary today, Crit. Anthropol., 32, 285-308, 2012.

Hall, D.: Land grabs, land control, and Southeast Asian crop booms, J. Peasant Stud., 38, 837-857, 2011.

Hall, D.: Primitive Accumulation, Accumulation by Dispossession and the Global Land Grab, Third World Q., 34, 1582-1604, 2013.

Haraway, D. J.: The companion species manifesto: Dogs, people, and significant otherness, Prickly Paradigm Press, Chicago, ISBN: 978-0971757585, 2003.

Haraway, D. J.: When species meet, University of Minnesota Press, Minneapolis, ISBN: 978-0816650453, 2008.

Harvey, D.: Justice, Nature and the Geography of Difference, Blackwell Publishers, Cambridge, MA, ISBN: 978-1557866813, 1996.

Harvey, D.: Spaces of capital: Towards a critical geography, Routledge, New York, ISBN: 978-0415932417, 2001.

Harvey, D.: The New Imperialism, Oxford University Press, Oxford, ISBN: 978-0191647758, 2003.

Holt-Giménez, E. and Altieri, M. A.: Agroecology, Food Sovereignty and the New Green Revolution, J. Sustain. Agr., 90-102, 2012.

Hustak, C. and Myers, N.: Involutionary Momentum: Affective Ecologies and the Sciences of Plant/Insect Encounters, Differences, 23, 74-118, 2013.

Ingram, M.: Fermentation, Rot, and Other Human-Microbial Performances, in: Knowing Nature: Conversations at the intersection of political ecology and science studies, Herausgeber: Goldman, M., Nadasdy, P., and Turner, M., University of Chicago Press, Chicago, 99-112, 2011.

Jansen, K.: The debate on food sovereignty theory: agrarian capitalism, dispossession and agroecology, J. Peasant Stud., 42, 1-20, 2014.

Kirksey, E. (Hrsg.): The Multispecies Salon, Duke University Press, Durham [NC], ISBN: 978-0822356103, 2014.

Kirksey, E. and Helmreich, S.: The Emergence of Multispecies Ethnography, Cult. Anthropol., 25, 545-576, 2010.

Kirksey, E., Shapiro, N., and Brodine, M.: Hope in blasted landscapes, Social Science Information, 52, 228-256, 2013.

Kohn, E.: How forests think: Toward an anthropology beyond the human, University of California Press, Berkeley, ISBN: 9780520956865, 2013.

Kohn, E.: Anthropology of Ontologies, Annu. Rev. Anthropol., 44 311-327, 2015.

Kueffer, C.: Ecological Novelty: Towards an Interdisciplinary Understanding of Ecological Change in the Anthropocene, in: Grounding global climate change: Contributions from the social and cultural sciences, Herausgeber: Greschke, H. und Tischler, J., Springer, Dordrecht, 19-37, 2015.

Latour, B.: Reassembling the social: An introduction to actornetwork-theory, Oxford University Press, Oxford, New York, ISBN: 978-0199256044, 2005.

Latour, B.: From multiculturalism to multinaturalism: What rules of method for the new socioscientific experiments?, Nature \& Culture, 6, 1-17, 2011. 
Latour, B.: Anthropology at the Time of the Anthropocene: a personal view of what is to be studied, Distinguished lecture, American Association of Anthropologists, Washington, 16 pp., 2014.

Leff, E.: The power-full distribution of knowledge in political ecology: a view from the South, in: The Routledge Handbook of Political Ecology, Herausgeber: Perreault, T., Bridge, G., and McCarthy, J., Routledge, New York, 64-75, 2015.

Levien, M.: From Primitive Accumulation to Regimes of Dispossession: Six Theses on India's Land Question, Econ. Polit. Weekly, 50, 146-157, 2015.

Levins, R. and Lewontin, R. C.: The dialectical biologist, Harvard University Press, Cambridge, Mass., ix, 303, ISBN: 0-67420283-X, 1985.

Li, T.: Practices of Assemblage and Community Forest Management, Econ. Soc., 36, 263-293, 2007.

Li, T. M.: To Make Live or Let Die? Rural Dispossession and the Protection of Surplus Populations, Antipode, 41, 66-93, 2010.

Lund, C.: Fragmented sovereignty: land reform and dispossession in Laos, J. Peasant Stud., 38, 885-905, 2011.

Lyons, K. M.: Soil Science, Development, and the "Elusive Nature" of Colombia's Amazonian Plains, The Journal of Latin American and Caribbean Anthropology, 19, 212-236, 2014.

Margulis, L.: Symbiotic planet: A new look at evolution, 1st ed, Basic Books, New York, ISBN: 978-0786724482, 1998.

Martínez-Torres, M. E. and Rosset, P.: Diálogo de saberes in La Vía Campesina: food sovereignty and agroecology, J. Peasant Stud., 41, 979-997, 2014.

Marx, K.: Das Kapital: Dritter Band: Der Gesamtprozeß der kapitalistischen Produktion, Dietz Verlag, Berlin, 1008 pp., 1968 [1867].

Mattissek, A. and Wiertz, T.: Materialität und Macht im Spiegel der Assemblage-Theorie: Erkundungen am Beispiel der Waldpolitik in Thailand, Geogr. Helv., 69, 157-169, doi:10.5194/gh-69-1572014, 2014.

McAfee, K.: Selling Nature to save It?: Biodiversity and Green Developmentalism, Environ. Plann. D, 17, 133-154, 1999.

McMichael, P.: The land grab and corporate food regime restructuring, J. Peasant Stud., 39, 681-701, 2012.

McMichael, P.: Historicizing food sovereignty, J. Peasant Stud., 41, 933-957, 2014a.

McMichael, P.: Rethinking Land Grab Ontology, Rural Sociol., 79, 34-55, 2014b.

Meek, D.: Agroecology and Radical Grassroots Movements' Evolving Moral Economies, Environ. Soc. Adv. Res., 5, 47-65, 2014.

Mignolo, W. D.: The Geopolitics of Knowledge and the Colonial Difference, S. Atl. Quart., 101, 57-97, 2002.

Moore, J. W.: Transcending the metabolic rift: a theory of crises in the capitalist world-ecology, J. Peasant Stud., 38, 1-46, 2011.

Moore, J. W.: Capitalism in the web of life: Ecology and the accumulation of capital, Verso, London, xi, ISBN: 978-1-78168-9028, 2015.

Münster, D.: Agrarian Alternatives: Agroecology, Food Sovereignty and the reworking of human-environmental relations in India, Rivista Degli Studi Orientali Nuova Serie, 88, 233-250, 2015 a.

Münster, D.: Farmers' Suicide and the Moral Economy of Agriculture: Victimhood, Voice, and Agro-Environmental Responsibility in South India, in: Suicide and agency: Anthropological perspectives on self-destruction, personhood and power, Heraus- geber: Broz, L. und Münster, D., Ashgate, Farnham, UK, Burlington, VT, 105-125, 2015b.

Münster, D.: Farmers' Suicides as Public Death: Politics, Agency and Statistics in a Suicide-Prone District (South India), Mod. Asian Stud., 49, 1580-1605, 2015c.

Münster, D.: Agro-ecological double movements? Zero Budget Natural Farming and alternative agricultures after the neoliberal crisis in Kerala, in: Critical Perspectives on Agrarian Transition: India in the Global Debate, Herausgeber: Mohanty, B. B., Routledge, New Delhi, 222-244, 2016.

Münster, D. and Münster, U.: Consuming the Forest in an Environment of Crisis: Nature Tourism, Forest Conservation and Neoliberal Agriculture in South India, Dev. Change, 43, 205-227, 2012.

Münster, D., Poerting, J., and Dame, J.: Agrarwirtschaft in Indien: Kleinbauern zwischen Krise und neuen Perspektiven, Geographische Rundschau, 1, 16-22, 2015.

Nüsser, M.: Ressourcennutzung und Umweltveränderung: MenschUmwelt-Beziehungen in peripheren Gebirgsräumen, in: $\mathrm{Hu}-$ manökologie: Ansätze zur Überwindung der Natur-KulturDichotomie, Herausgeber: Meusburger, P. und Schwan, T., Franz Steiner Verlag, Stuttgart, 327-342, 2003.

Ouma, S.: Situating global finance in the Land Rush Debate: A critical review, Geoforum, 57, 162-166, 2014.

Ouma, S.: Assembling Export Markets: The Making and Unmaking of Global Food Connections in West Africa, Wiley-Blackwell, New York, ISBN: 978-1118632581, 2015.

Palekar, S.: The Philosophy Of Spiritual Farming: Zero Budget of Natural Farming - Part 1, 5th revised renewal edition, Zero Budget Natural Farming Research, Development \& Extension Movement, Amravati (Maharashtra), 215 pp., 2010.

Patel, R.: What does food sovereignty look like?, J. Peasant Stud., 36, 663-706, 2009.

Paxson, H.: Microbiopolitics, in: The Multispecies Salon, Herausgeber: Kirksey, E., Duke University Press, Durham NC, 115121, 2014.

Peet, R., Robbins, P., and Watts, M. (Hrsg.): Global Political Ecology, Routledge, London, ISBN: 978-0415548144, 2011.

Peluso, N. L. and Lund, C.: New Frontiers of Land Control: Introduction, J. Peasant Stud., 38, 667-681, 2011.

Poerting, J.: Who controls the land in certified organic farming?: Discerning the divide between de jure and de facto land control, in: Aktuelle Forschungsbeiträge zu Südasien: 5. Jahretagung des AK Südasien, 23./24. Januar 2015, Herausgeber: Poerting, J. und Keck, M., Göttingen, 29-32, 2015.

Poerting, J.: Soziale Innovation oder Business as Usual? Zertifizierte Bio-Landwirtschaft in Nordpakistan, Geogr. Z., 104, im Erscheinen, 2016.

Polanyi, K.: The great transformation: Politische und ökonomische Ursprünge von Gesellschaften und Wirtschaftssystemen, 3. Aufl., Suhrkamp, Frankfurt (Main), ISBN: 978-3-518-27860-4, 1995 [1944].

Pollan, M.: The botany of desire: A plant's-eye view of the world, Random House trade pbk. ed., Random House, New York, ISBN: 978-0375501296, 2002.

Puig de la Bellacasa, M.: Making time for soil: Technoscientific futurity and the pace of care, Soc. Stud. Sci., 45, 691-716, 2015. 
Schmidt, M.: Interdependencies and reciprocity of private and common property resources in the Central Karakorum, Erdkunde, 58, 316-330, 2004.

Schneider, M. and McMichael, P.: Deepening, and repairing, the metabolic rift, J. Peasant Stud., 37, 461-484, 2010.

Shiva, V., Emani, A., and Jafri, A. H.: Globalisation and Threat to Seed Security: Case of Transgenic Cotton Trials in India, Econ. Polit. Weekly, 34, 601-613, 1999.

Singh, N.: The affective labor of growing forests and the becoming of environmental subjects: Rethinking environmentality in Odisha, India, Geoforum, 47, 189-198, 2013.

Star, S. L. and Griesemer, J. R.: Institutional Ecology, "Translations" and Boundary Objects: Amateurs and Professionals in Berkeley's Museum of Vertebrate Zoology, 1907-39, Soc. Stud. Sci., 387-420, 1989.

Steffen, W., Richardson, K., Rockstrom, J., Cornell, S. E., Fetzer, I., Bennett, E. M., Biggs, R., Carpenter, S. R., Vries, W. de, de Wit, C. A., Folke, C., Gerten, D., Heinke, J., Mace, G. M., Persson, L. M., Ramanathan, V., Reyers, B., and Sorlin, S.: Planetary boundaries: Guiding human development on a changing planet, Science, 347, 736-746, 2015.

Stengers, I.: Cosmopolitics I, University of Minnesota Press, Minneapolis, ISBN: 978-0-8166-5687-5, 2010.

Swanson, H. A., Bubandt, N., and Tsing, A.: Less Than One But More Than Many: Anthropocene as Science Fiction and Scholarship-in-the-Making, Environ. Soc. Adv. Res., 6, 149166, 2015.

Swyngedouw, E.: Modernity and Hybridity: Nature, Regeneracionismo, and the Production of the Spanish Waterscape, 18901930, Ann. Assoc. Am. Geogr., 89, 443-465, 1999.
Tsing, A.: More-than-Human Sociality: A Call for Critical Description, in: Anthropology and nature, Herausgeber: Hastrup, K., Routledge, New York, 27-42, 2014.

Vandermeer, J. H.: Reconstructing biology: Genetics and ecology in the new world order, Wiley, New York, ISBN: 978-0471109174, 1996.

Viveiros de Castro, E.: Cosmological Perspectivism in Amazonia and Elsewhere, HAU, Manchester, ISBN: 20494769, 2012.

Viveiros de Castro, E.: Who is afraid of the ontological wolf?: some comments on an ongoing anthropological debate, The Cambridge Journal of Anthropology, 33, 2-17, 2015.

Walker, P. A.: Political ecology: where is the ecology?, Prog. Hum. Geogr., 29, 73-82, 2005.

Weichhart, P.: Gesellschaftlicher Metabolismus und Action Settings: Die Verknüpfung von Sach- und Sozialstrukturen im alltagsweltlichen Handeln, in: Humanökologie: Ansätze zur Überwindung der Natur-Kultur-Dichotomie, Herausgeber: Meusburger, P. und Schwan, T., Franz Steiner Verlag, Stuttgart, 15-44, 2003.

Weichhart, P.: Der Kieler Geographentag 1969 - Modernisierungsschub, Mythos, Paradigmenwandel oder vergessene Geschichte?, Geogr. Helv., 71, 7-13, doi:10.5194/gh-71-7-2016, 2016.

Weis, T.: The Accelerating Biophysical Contradictions of Industrial Capitalist Agriculture, J. Agrar. Change, 10, 315-341, 2010.

Whatmore, S.: Hybrid Geographies. Natures cultures spaces, Sage, London, ISBN: 978-1446240267, 2002. 